\title{
Infrared Spectroscopy and Molecules in Circumstellar Envelopes
}

\author{
Kenneth H. Hinkle
}

Kitt Peak National Observatory, National Optical Astronomy Observatories ${ }^{\star}$, Tucson, Arizona, USA 85719

\section{Introduction}

During the last two decades there has been a tremendous renewal of interest in the circumstellar environment of evolved stars. This renewal has been mainly driven by sensitive microwave observing techniques. Microwave spectroscopy has resulted in a number of fundamental discoveries in circumstellar shells, including the discovery of maser lines and complex circumstellar chemistry. Historically, interest in circumstellar shells originates from a desire to understand the massloss process. It has been known for at least 50 years that circumstellar gas is the origin of interstellar gas and dust. As such the circumstellar shell becomes the pathway through which elements created in the interior of the central star enrich galactic abundances. High resolution spatial and spectral techniques and an ever improving knowledge of stellar evolution has resulted in an increased but still incomplete understanding of the mass-loss process.

There is sufficient space here only to review a small subtopic in this research area. This report is divided into four parts. First, the structure and dynamics of circumstellar shells will be briefly reviewed. Second, the same will be done for circumstellar chemistry. Third, the impact of infrared observations will be discussed in depth. Finally, the future of infrared spectroscopy will be discussed. The review will not concentrate on absorption coefficients for the molecules discussed. Jørgensen (1992) presents an excellent review of this material.

\section{Circumstellar Shells}

The evolved late-type stars are divided into two basic groups depending on whether the oxygen or carbon is the most abundant. The $\mathrm{C} / \mathrm{O}$ ratio plays a critical role in the chemistry of the stellar atmosphere (Tsuji 1964, 1973; Scalo \& Ross 1976). Stars with $\mathrm{C}<\mathrm{O}$ are referred to as oxygen-rich or M-type while those with $\mathrm{C}>\mathrm{O}$ are carbon-rich or C-type. Notably for chemistry the relative

\footnotetext{
* Operated by the Association of Universities for Research in Astronomy, Inc. under cooperative agreement with the National Science Foundation.
} 
$\mathrm{C} / \mathrm{O}$ abundance is of primary importance with the astrophysically important $\mathrm{C} / \mathrm{H}$ and $\mathrm{O} / \mathrm{H}$ abundances of secondary importance.

The simplest model of the circumstellar shell starts with a central star with a relatively thin (compared to the stellar radius), gravitationally bound photosphere. The photosphere terminates in a chromosphere or perhaps in a cool extension to the photosphere. This region is non-expanding and significant in size, extending several stellar radii above the photosphere, and terminates at the beginning of the expanding circumstellar envelope. This classical view and the evidence supporting it is discussed in reviews by Zuckerman (1980), Dupree (1986), and Goldberg (1987) among others. The great utility of simple models can be seen in their ability to model microwave line profiles and derive mass-loss rates assuming only that (1) the envelope is spherically symmetric, (2) the massloss rate and the terminal velocity are constant around the envelope, implying density proportional to $\mathrm{r}^{-2}$, and (3) local turbulent or thermal motions are much less than the terminal velocity (see Kastner 1992 and references therein).

For circumstellar shells of cooler giant stars, which are the primary concern of this review, three principal features characterize the circumstellar shell (Glassgold \& Huggins 1987). (1) The terminal wind speeds are small compared to the stellar escape velocity and are typically on the order of $10 \mathrm{~km} \mathrm{~s}^{-1}$. (2) The circumstellar envelope is much larger than the stellar radius, $R_{\text {shell }} / R_{\text {star }} \sim$ $10^{3}$. The implied variation in physical conditions is enormous. (3) The mass-loss rates are large, typically in the range $10^{-5}$ to $10^{-7} \mathrm{M}_{\odot} \mathrm{yr}^{-1}$. Since the stellar masses can be as small as $1-2 \mathrm{M}_{\odot}$, the time scale for this stellar evolutionary stage is relatively short. It is generally accepted that the outflow in the expanding circumstellar envelope is driven by radiation pressure on dust (Jones et al. 1981; Dominik et al. 1990). For carbon-rich dust, infrared spatial interferometry of prototypical sources has set the dust formation temperature at around $1300 \mathrm{~K}$ (Danchi et al. 1990). Oxygen-rich dust has a condensation temperature of about this value.

While the simple model must be basically correct since it provides reasonable matches to many kinds of observations, there has been observational evidence for some time that the actual situation is more complex. Asymmetries in the circumstellar shell of the otherwise normal M supergiant $\alpha$ Ori are well documented (e.g. Bernat \& Lambert 1976; Mauron et al. 1984; Hebden et al. 1987). For a sample of $\mathbf{M}$ giants, Bernat et al. (1979) and Bernat (1981) found multiple velocity components in the profiles of infrared $\mathrm{CO}$ lines seen in absorption against the stellar photosphere. The velocity and excitation temperature of these components are derivable. For some stars velocity increased as excitation temperature decreased. This can be explained as radiation pressure changes on grains as different circumstellar gases freeze onto grains (Keady et al. 1988). However, for a few stars velocity and excitation temperature were not related. This can be explained by discrete or time varying mass-loss events (Jura 1986; Olofsson et al. 1993). The extreme case of $\mu$ Cep (Bernat 1981) may require clumping in the circumstellar shell. VLBI maps of SiO masers (Moran et al. 1979) show that the $\mathrm{SiO}$ maser results from a large number of spots rather than the entire 
circumstellar shell. Alcock \& Ross (1986) have shown that the masers form in 'dense cloudlets' and not in the spherically expanding circumstellar wind and suggest that the density enhanced clouds are formed by the ejection process from the photosphere. Ultimately, the circumstellar shell of a red giant becomes the expanding shell of a planetary nebula. Planetary nebulae are marked more by their deviation from spherical symmetry than their adherence to it.

While far more mass is typically in the gas than the dust (i.e. Harvey et al. 1991 estimate $\mathbf{M}_{\text {gas }} / \mathbf{M}_{\text {dust }} \sim 600$ in IRC+10216), the dust is an important consideration for the spectroscopist. The dust re-radiates absorbed radiation from the photosphere of the star into the infrared. A large class of objects exists where the dust has enough optical depth to obscure the photosphere. These objects are of special interest for spectroscopy of circumstellar lines. In normal stars the photosphere contributes a large number of lines to the spectrum throughout the infrared. In stars obscured by optically thick circumstellar dust only the circumstellar lines are seen. The $\mathrm{C} / \mathrm{O}$ ratio determines the composition of the dust as well as the gas, and the dust composition impacts the circumstellar structure. In the oxygen-rich case, the grains are oxides of refractory elements especially silicon, while in the carbon-rich case, the grains are largely carbon combined with refractory elements (Turner 1991).

This review uses the terms column density and abundance frequently. The abundance is the number of molecules per unit volume relative to the number of hydrogen molecules per unit volume. The density decreases with radius as $\mathrm{r}^{-2}$; by referencing the abundance to a stable molecule this effect is removed. The column density is the total number of molecules per square centimeter along the line of sight to the star. Many classes of molecules do not exist throughout the circumstellar shell but exist only through a portion of the circumstellar radius. Thus for an observed molecule, dividing the observed column density by the column density of molecular hydrogen does not necessarily produce a physically meaningful abundance. While this is now well understood, incorrect abundances can be found in early papers. Perhaps more meaningful for molecules created in the circumstellar shell is the peak radial abundance which is the largest value of the abundance at any point along a radius from the central star.

To determine the peak radial abundance of a molecule, line profiles must be synthesized using a model of the circumstellar shell that specifies the run of temperature, velocity, and density with radius. The determination of a peak radial abundance is possible because the physical conditions may be uniquely assigned by radius. The run of physical conditions with radius results in a distinctive line shape for each spectral line as well as a characteristic change of line strength with rotational energy. Modeling the line profile as well as the change of profile and equivalent width with rotational energy allows the modeler to determine the radial distribution of the molecule in the model. The circumstellar model can be determined semiempirically from lines of a molecule like CO, which has the stability to exist throughout the vast range of physical conditions from the cool star photosphere to the outer circumstellar radius (Keady et al. 1988). Since infrared spectroscopy typically probes the inner circumstellar shell $(10 \lesssim R \lesssim 1000$ 
$\left.R_{*}\right)$ and microwave spectroscopy probes outer regions $\left(R_{\approx} \geq 1000 R_{*}\right)$, abundances derived from different techniques may be somewhat different and there is always uncertainty as to whether this reflects measurement differences or circumstellar chemistry (Keady \& Hinkle 1988).

\section{Circumstellar Molecules and Chemistry}

The chemistry of circumstellar envelopes is a topic of considerable current interest because of both theoretical and observational challenges. Several reviews appear in the recent literature (Omont 1985; Glassgold \& Huggins 1987). The circumstellar envelope challenges the theorist because the chemistry is timedependent as the material is carried away from the star through regions with widely differing physical conditions. The simplest chemical model is the 'freeze out' model. In this model thermal equilibrium applies close to the central star. As the density of the circumstellar gas decreases the temperature drops below some critical value at which the chemical reactions stop and the abundances are 'frozen'. Freeze out models are presented by McCabe et al. (1979) and Lafont et al. (1982). In general these models work well for simple CNO molecules but fail for refractory compounds, chain molecules, and radicals. A more refined model includes chemical reactions in the circumstellar shell driven by photodissociation and photoionization. These processes are driven by interstellar ultraviolet, chromospheric ultraviolet (in unobscured circumstellar envelopes), and cosmic rays. Ions produced through photoionization, for instance $\mathrm{C}_{2} \mathrm{H}_{2}^{+}$, can lead to rapid reactions which build large molecules (Nejad et al. 1984; Glassgold et al. 1986). This model is in agreement with spatially resolved microwave images of circumstellar shells which show that complex molecules exist in the outer envelope but not near the star (e.g. Bieging \& Tafalla 1993). Models are just appearing which include reactions that take place in or on grains (Brown et al. 1988). Glassgold \& Huggins (1987) note that shocks in pulsating stars could also drive chemical processes.

A current list of molecules detected using microwave and infrared techniques can be found in Olofsson (1992). Much of the observational work on the chemistry in circumstellar envelopes has been done on the carbon-rich envelope around the bright, obscured carbon star IRC+10216. Interestingly IRC+10216 is such a rich source and such a bright object that a number of the molecules detected are seen only in this source. Detailed chemical models exist for IRC+10216 (e.g. Glassgold et al. 1986; Nejad \& Millar 1987; Howe \& Millar 1990). The models include the photodissociation and photoionization of a large number of molecules of photospheric origin. The photodissociation and photoionization chemistry of the most common molecules, $\mathrm{H}_{2}, \mathrm{CO}, \mathrm{HCN}, \mathrm{C}_{2} \mathrm{H}_{2}$, is quite complex. Of particular interest is the photoionization of $\mathrm{C}_{2} \mathrm{H}_{2}$ which leads to the formation of complex molecules including carbon chains bearing hydrocarbons. Frenklach \& Feigelson (1989) have shown that acetylene chemistry can produce benzene and ultimately polycyclic aromatic hydrocarbons.

Work on the chemistry of oxygen-rich circumstellar shells is less developed 
than for carbon-rich circumstellar shells. This stems from two causes. First, there is no highly obscured, bright star that would be an oxygen-rich analogy to the carbon-rich star IRC +10216 . Second, in oxygen-rich circumstellar shells carbon is mainly associated into $\mathrm{CO}$ and the chemistry has not been considered as interesting. Recently there has been increased interest in oxygen-rich circumstellar shells as a result of the discovery of post-AGB, pre-planetary nebula objects in the form of molecule-rich bipolar nebulae (e.g. OH231.8+4.2 [Morris et al. 1987]).

Mamon et al. (1987) present a model for photochemistry and molecular ions in oxygen-rich circumstellar envelopes. This paper is restricted to photochains associated with $\mathrm{CO}, \mathrm{H}_{2} \mathrm{O}$, and $\mathrm{N}_{2}$. It was shown that the molecular ions $\mathrm{H}_{3} \mathrm{O}^{+}$, $\mathrm{HCO}^{+}$and $\mathrm{H}_{3}^{+}$all play an important role in the photochemistry. In particular, the polar ions $\mathrm{H}_{3} \mathrm{O}^{+}$and $\mathrm{HCO}^{+}$are predicted to have a large peak abundance. Omont et al. (1993) present observations and discussion of sulfur compounds in oxygen-rich circumstellar envelopes. References to the gas phase chemistry are given by Omont et al. (1993).

\section{The Infrared spectrum}

Microwave spectroscopy has been shown to be tremendously successful at detecting molecules in circumstellar and interstellar environments. Infrared spectroscopy is a complementary technique. The infrared spectrum contains stretch and bending vibration transitions for molecules made up from combinations of the astrophysically important CNO group atoms with themselves and hydrogen. Figure 1 adapted from Allamandola (1984) summarizes the spectral locations of the most important transitions. In addition, the infrared has permitted rotationvibration transitions of symmetric molecules which have no permitted pure rotation transitions in the microwave. Historically, a critical role for the infrared has been in establishing the abundances of symmetric molecules like $\mathrm{H}_{2}, \mathrm{C}_{2} \mathrm{H}_{2}$, and $\mathrm{CH}_{4}$. While these symmetric molecules also have permitted electronic transitions, the flux from cool sources is so low in the ultraviolet where most of these transitions occur that they will be mainly unobservable. (A few electronic transitions occur in the infrared, see Bernath 1992.) In addition, opacity caused by interstellar and circumstellar dust is large in the blue but at a minimum in the $2 \mu \mathrm{m}$ infrared. In circumstellar shells, the infrared may be used to probe regions of the circumstellar shell closer to the star than can be seen in the microwave. Of the molecules previously detected in the microwave most have transitions in parts of the infrared not blocked by telluric absorption. Many of these transitions have large transition moments but have not been exploited because the relatively small column densities and/or low fluxes of the sources have exceeded the capabilities of existing infrared detectors.

\subsection{Carbon-Rich Circumstellar Shells}

If $\mathrm{C}<\mathrm{O}$, the $\mathrm{CO}$ molecule bonds all the available carbon atoms. However, if $\mathrm{C}>\mathrm{O}$, extra carbon atoms remain to combine with hydrogen, nitrogen and other 


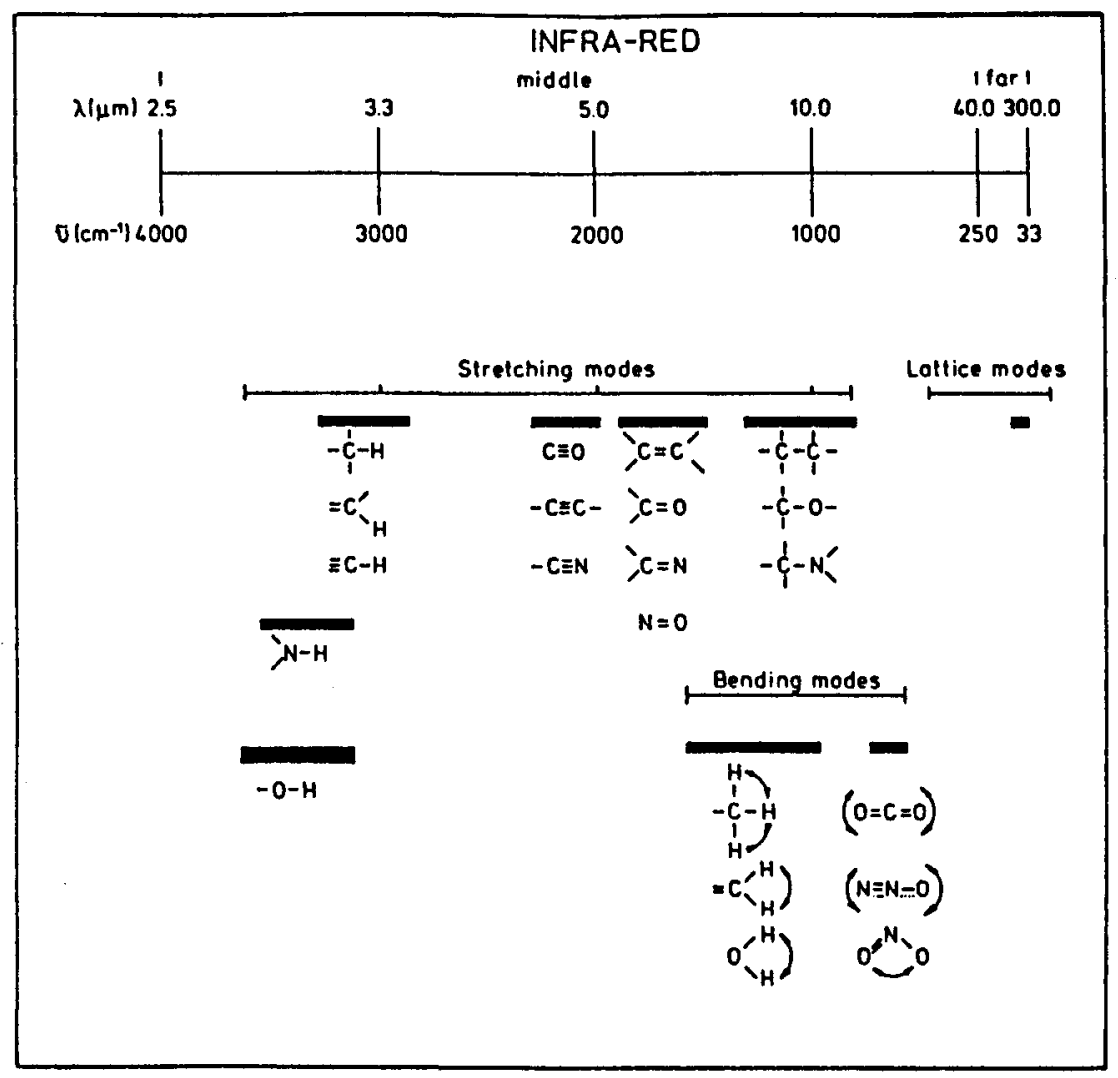

Fig. 1. Spectral locations of HCNO group molecular stretch and bend transitions in the infrared. In a gas, these transitions consist of a number of rotation-vibration lines. Figure from Allamandola 1984 as adapted by Genzel 1992

carbon atoms, as well as less abundant elements. As can be seen from Figure 1 the rotation-vibration spectra of these molecules are observable in the $3-12 \mu \mathrm{m}$ infrared.

The most abundant circumstellar molecule is $\mathrm{H}_{2}$, the principal constituent of circumstellar shells. Typically $\mathrm{H}_{2}$ is $10^{3}$ times more abundant than any other molecule. However, the homonuclear $\mathrm{H}_{2}$ molecule has only a weak quadrupole spectrum. Oscillator strengths are $10^{6}$ times smaller than the oscillator strengths of typical diatomic molecules. The factor of $10^{6}$ more than compensates for the large abundance of $\mathrm{H}_{2}$, so sources which have exceeding strong spectral lines from typical diatomic molecules need not have detectable $\mathrm{H}_{2}$ lines. Weak circumstellar absorption lines from the 1-0 $S(0)$ and $S(1)$ (i.e. $v^{\prime \prime}=0, J^{\prime \prime}=0 \rightarrow v^{\prime}=1, J^{\prime}=2$ and $\left.v^{\prime \prime}=0, \mathrm{~J} "=1 \rightarrow v^{\prime}=1, J "=3\right)$ lines have been detected in the $2 \mu \mathrm{m}$ spectra of Mira variables (Hinkle et al. 1994a) and the 1-0 S(1) line has been detected in the spectrum of IRC +10216 (Hinkle et al. 1994b). Keady \& Ridgway (1993) provide 
models indicating that this line is formed in the inner, slowly expanding part of the circumstellar shell.

$\mathrm{CO}$ is ubiquitous to circumstellar and interstellar environments and as opposed to $\mathrm{H}_{2}$ is easily detectable spectroscopically. $\mathrm{CO}$ rotation-vibration lines of circumstellar origin can be observed in the $4.6 \mu \mathrm{m}$ spectra of typical M-type and C-type stars (Bernat 1981). The obscured carbon-star IRC+10216 presents the best modeled example. The CO spectrum was explored in detail by Keady et al. (1988) who used the CO line profile to develop a semiempirical model atmosphere. The infrared provides information on sections of the circumstellar shell much closer to the star than can be observed in microwave CO. In the infrared, the individual $\mathrm{CO}$ rotation-vibration lines are seen in absorption against a continuum formed by thermal emission from the dust. Emission resulting from resonant scattering in the CO lines can be seen in the profiles of the CO $\Delta \mathrm{v}=1$ low excitation lines as well as in off source spectra (Sahai \& Wannier 1985). CO line profiles in IRC+10216 clearly show multiple velocity components. These components appear to result from discrete changes in the radiation pressure on grains and have been attributed to the freezing of circumstellar gases on grain mantles. The IRC +10216 low excitation $\mathrm{CO}$ fundamental lines are optically thick and ${ }^{12} \mathrm{C}^{16} \mathrm{O},{ }^{13} \mathrm{C}^{16} \mathrm{O},{ }^{12} \mathrm{C}^{17} \mathrm{O}$, and ${ }^{12} \mathrm{C}^{18} \mathrm{O}$ are all easily detected.

The next most abundant molecule in carbon-rich circumstellar shells is generally acetylene. $\mathrm{C}_{2} \mathrm{H}_{2}$ has a rich spectrum, with lines detected in IRC+10216 from 2.5 through $12 \mu \mathrm{m}$. With such a wide wavelength range, $\mathrm{C}_{2} \mathrm{H}_{2}$ is a useful probe of circumstellar structure, as discussed by Keady \& Ridgway (1993). From the point of view of opacities the most remarkable feature of acetylene is the 3 $\mu \mathrm{m}$ band. This band results from the $\mathrm{C}_{2} \mathrm{H}_{2}$ and $\mathrm{HCN} \mathrm{C}-\mathrm{H}$ stretch (Ridgway et al. 1978). In IRC +10216 the strongest lines in this band are optically thick and the line spacing is dense. Sufficient lines are present that other much less abundant molecules could contribute to the band but would be undetectable. The ${ }^{13} \mathrm{C}^{12} \mathrm{CH}_{2}$ isotopic lines are easily detected, in spite of the fact that IRC+10216 has a ${ }^{12} \mathrm{C} /{ }^{13} \mathrm{C}$ ratio of $\sim 40$ (Wannier \& Sahai 1987 ; Keady et al. 1988). In IRC+10216 Keady \& Hinkle (1988) find evidence that the $\mathrm{C}_{2} \mathrm{H}_{2}$ abundance decreases by a factor of 5 between 100 and $1000 R_{*}$. This suggests that the acetylene abundance is reduced through interactions with grain surfaces.

$\mathrm{HCN}$ has approximately the same abundance as $\mathrm{C}_{2} \mathrm{H}_{2}$ in the circumstellar shell of IRC+10216. Wiedemann et al. (1991) find an inner shell abundance of $4 \times 10^{-5}$ for HCN and a column density of $7 \times 10^{18} \mathrm{~cm}^{-2}$ while Keady \& Hinkle (1988) measured an inner shell abundance of $5 \times 10^{-5}$ for $\mathrm{C}_{2} \mathrm{H}_{2}$ with a column density of $2 \times 10^{18} \mathrm{~cm}^{-2}$. As is the case for $\mathrm{H}_{2}, \mathrm{CO}$ and $\mathrm{C}_{2} \mathrm{H}_{2}$, the circumstellar HCN line profiles in IRC+10216 show multiple velocity components. Since these result from accelerations known from the $\mathrm{CO}$ to occur fairly close to the central star, they are the infrared spectral signature of a molecule of photospheric origin. The photospheric origin of HCN is not surprising, since HCN has been known for some time to be an important opacity contributor to the upper photosphere of unobscured, cool carbon stars (Jørgensen 1990).

The remaining molecules detected in the infrared all have peak radial abun- 
dances an order of magnitude, or more, smaller than the above major contributors. These will be divided into classes according to their circumstellar chemistry. Figure 2 taken from Keady \& Ridgway (1993) illustrates the relative molecular abundances in the prototypical carbon rich circumstellar shell around IRC +10216 . The first group are molecules of photospheric origin. These include $\mathrm{SiO}$ and CS. In IRC+10216, Keady and Ridgway (1993) report that CS shows evidence of decreasing column density with radius in the range observed by the infrared. In addition, the infrared CS abundance is an order of magnitude larger than the microwave CS abundance. Since the CS abundance appears to change from 10 to $1000 R_{*}$, an interval where photolysis is not important, the depletion must be due to absorption on the grain surfaces (Keady \& Ridgway 1993). On the other hand, infrared and microwave abundances of $\mathrm{SiO}$ in IRC+10216 are fairly well matched, implying a constant abundance out to the radius where photolysis occurs.

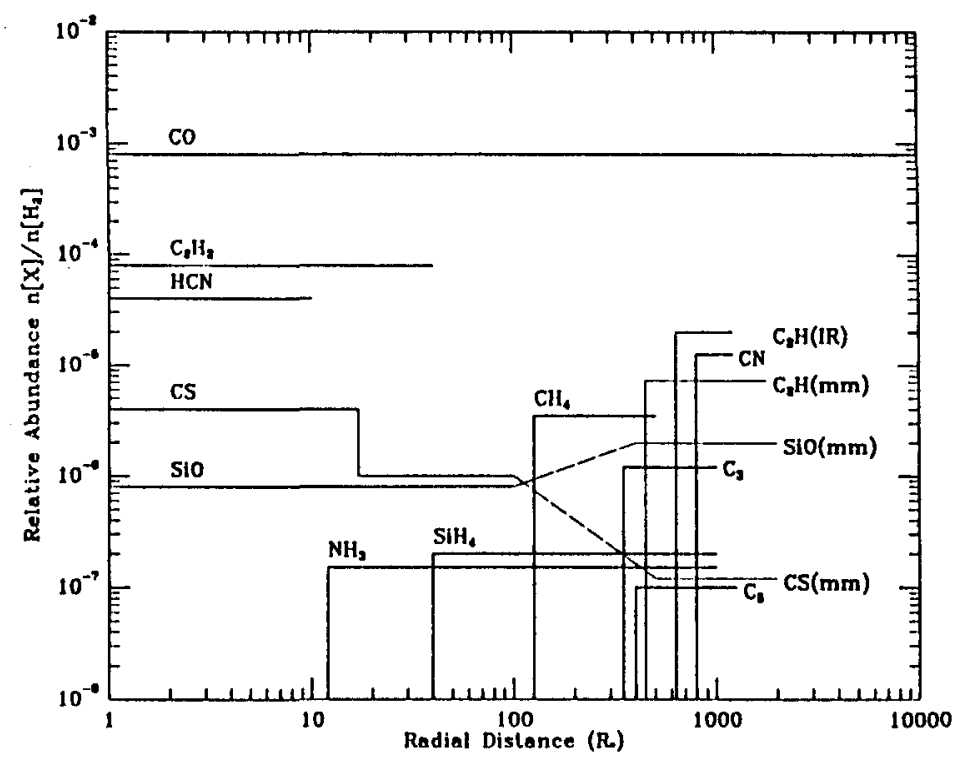

Fig. 2. Radial abundances in IRC +10216 for molecules detected in infrared spectra. The source of abundances are labeled for outer shell molecules with abundances determined from both infrared (IR) and millimeter wave (mm) observations. All abundances at $R<100 R_{*}$ are infrared. From Keady and Ridgway 1993

Ammonia $\left(\mathrm{NH}_{3}\right)$, silane $\left(\mathrm{SiH}_{4}\right)$, and methane $\left(\mathrm{CH}_{4}\right)$, form a class of molecules created in the mid-circumstellar shell through grain-surface chemistry. The geometric similarity of the three molecules is notable. Infrared line profiles for these three molecules, unlike profiles for $\mathrm{CO}$ or $\mathrm{C}_{2} \mathrm{H}_{2}$ lines, do not have a contribution from the circumstellar gas at velocities close to the star. Detailed modeling by Keady \& Ridgway (1993) shows that the lines from each molecule can be best 
fit by radial abundances that have a cut off at an inner radius relatively close to the star compared to the usual case for molecules detected in the microwave. In the case of $\mathrm{NH}_{3}$ this radius is $\sim 20 \mathrm{R}_{*}$, for $\mathrm{SiH}_{4} \sim 40 \mathrm{R}_{*}$, and for $\mathrm{CH}_{4} \sim 100$ $R_{*}$. Keady \& Ridgway (1993) note that the production of these gases in the circumstellar shell occurs near radii where the gas is accelerated. They point to a link between grain surface chemical changes and changing photon pressure on the grains. Photolysis clearly does not play a role in forming these molecules since the circumstellar shell is well shielded from UV where they are formed.

The above evidence points to an origin of these molecules on the grain surface. Detections of these molecules and the knowledge that they were formed in the mid-circumstellar shell have been known for some time $\left(\mathrm{CH}_{4}\right.$ : Clegg et al. 1982; $\mathrm{NH}_{3}$ : Betz et al. 1979; $\mathrm{SiH}_{4}$ :Goldhaber \& Betz 1984). However, progress in this area has been motivated by dense interstellar clouds. To match interstellar $\mathrm{NH}_{3}$ abundances Brown et al. (1988) and others proposed that $\mathrm{CH}_{4}$ and $\mathrm{NH}_{3}$ form by hydrogenation of $\mathrm{C}$ and $\mathrm{N}$ on grain mantles. Presumably a similar mechanism applies to $\mathrm{SiH}_{4}$. Lacy et al. (1991) found observational evidence that $\mathrm{CH}_{4}$ is made in molecular cloud grain mantles. Charnley \& Miller proposed a mechanism for $\mathrm{H}_{2} \mathrm{O}$ also to be formed on grain mantles but this hypothesis is probably not testable due to the already high water abundance in oxygen-rich environments.

A possibly related molecule is ethylene $\left(\mathrm{C}_{2} \mathrm{H}_{4}\right)$. Goldhaber et al. (1987) report that ethylene has an average rotation temperature of $100 \mathrm{~K}$ and a column density of $4 \times 10^{15} \mathrm{~cm}^{-2}$. However, the rotational levels of ethylene did not seem to be distributed in LTE as is the case for the above three molecules. The data were not of high $\mathrm{S} / \mathrm{N}$ and additional infrared observations of ethylene would be of interest.

$\mathrm{C}_{2} \mathrm{H}$ and $\mathrm{CN}$ are two photolysis molecules that have been detected in the infrared spectrum of IRC +10216 . Both molecules have rotational temperatures on the order of $10 \mathrm{~K}$ and column densities near $3 \times 10^{15}$. The $\mathrm{CN}$ result is in good agreement with $\mathrm{CN}$ microwave measurements and with formation from photolysis of $\mathrm{HCN}$ (Wiedemann et al. 1991). $\mathrm{C}_{2} \mathrm{H}$ infrared and microwave results are again in agreement (Keady \& Hinkle 1988). The parent molecule for $\mathrm{C}_{2} \mathrm{H}$ is $\mathrm{C}_{2} \mathrm{H}_{2}$.

The naked carbon chain molecules $\left(\mathrm{C}_{3}\right.$ and $\left.\mathrm{C}_{5}\right)$ detected in the infrared (Hinkle et al. 1988; Bernath et al. 1989) have an unknown origin. The line profiles of both of these molecules clearly indicate that they are formed in the circumstellar shell. Both $C_{3}$ and $C_{5}$ have a respectably large abundance $\left(\sim 10^{-6}\right.$ for $C_{3}$ and $\sim 10^{-7}$ for $C_{5}$ ). The column density of $C_{5}$ is $1 \times 10^{14} \mathrm{~cm}^{-2}$. No parent molecule with sufficient abundance has been identified. The excitation temperatures of both molecules are $\sim 40 \mathrm{~K}$. The most likely suggestion at this time is that these molecules result from photolysis of the carbonaceous grains. This is a distinct process from the grain surface chemistry discussed above.

We have searched for a number of other naked carbon chains up to $\mathrm{C}_{60}$. The odd numbered chains may be less reactive than the even chains and hence more easily detectable (Kroto et al. 1987). However, at this time the detection of odd numbered chains has mainly to do with the infrared spectra. $\mathrm{C}_{4}$ has its strongest 
infrared transition at $1549 \mathrm{~cm}^{-1}$ where it is blocked from observation by telluric water vapor. The odd numbered chains $\mathrm{C}_{3}, \mathrm{C}_{5}, \mathrm{C}_{7}, \mathrm{C}_{9}$ occur in atmospheric windows. An upper limit of $<2 \times 10^{13} \mathrm{~cm}^{-2}$ has been set for the $\mathrm{C}_{7}$ column density (Hinkle \& Bernath 1992).

The fullerenes, which are larger members of the naked carbon chain family deserve a special note. These molecules turn out to be difficult to detect spectroscopically. The $\mathrm{C}_{60}$ molecule has a high symmetry which results in only four vibration modes being infrared active. These are the four modes reported by Kratschmer et al. (1990): 528, 577, 1183 , and $1429 \mathrm{~cm}^{-1}$. Of these four only 1183 $\mathrm{cm}^{-1}$ is reasonably placed in the spectrum to observe astronomically. Frum et al. (1991) found that the $1183 \mathrm{~cm}^{-1}$ band in the solid phase was at $1169.1 \mathrm{~cm}^{-1}$ in the gas phase. The rotational structure of this transition is so closely spaced as to be unresolvable. In addition, there is no sharp, distinctive Q-branch which makes detection of the $1169 \mathrm{~cm}^{-1}$ band difficult. While we could not detect this band in IRC+10216, this could be due to hydrogenation in this source and it might be of interest to try observing a yet more carbon-rich source.

Polycyclic aromatic hydrocarbons (PAHs), the family of ring molecules of which benzene is the simplist, are the suspected carriers of a number of widely observed infrared emission features. A primary source of PAHs may be mass-losing carbons stars (Latter 1991). Detailed chemical reaction networks developed by Frenklach \& Feigelson (1989) indicate that PAH can be produced from acetylene with yields in excess of $1 \%$. The above mentioned depletion of acetylene with increasing radius in the circumstellar shell of $\mathrm{IRC}+10216$ could reflect this process (Latter 1991). At a 1\% yield, the PAH column density in IRC +10216 would be on the order of $10^{16}$. Latter (1991) has suggested that PAHs may be taken up by grains shortly after the PAH molecule is created, only to be released later in the interstellar medium. Even if the PAHs remain in the gas phase they will be nearly impossible to detect in a circumstellar shell. As is well known, the PAHs include a large number of different molecules (see Léger et al. 1987). Microwave spectroscopy is impossible because the rotational spectra are extremely complex to the confusion limit and individual molecules in the group are not necessarily very abundant. The $\mathrm{CH}$ stretch frequency depends on the bonding and on the side groups attached, resulting in a PAH CH stretch at $3050 \mathrm{~cm}^{-1}$ while the acetylene $\mathrm{CH}$ stretch is at $3300 \mathrm{~cm}^{-1}$. However, the prognosis for detection by high resolution techniques (i.e. line resolved spectroscopy) in the infrared is the same as in the microwave for basically the same reasons. In the interstellar medium relatively low concentrations of PAHs can be seen excited by ultraviolet radiation. In circumstellar shells this excitation mechanism is not active due to UV shielding.

In sources that become sufficiently cold before photoionization and photodissociation commence, carbon compounds form ices. CO has a sublimation temperature of 15-20 K under astrophysical conditions (Tielens et al. 1991). When $\mathrm{CO}$ goes into the solid state rotation is no longer allowed, thus the $\mathrm{R}$ and $\mathbf{P}$ branches disappear. However, a pure vibration transition, the $\mathrm{Q}$ branch which is not permitted in the gas phase, is allowed in the solid phase. The $\mathrm{Q}$ branch 
appears in the spectrum at about the location of the band origin. Non-polar compounds all have sublimation temperatures below that of polar compounds. Thus the common carbon-rich ices have sublimation temperatures considerably below that of water ice (on the order of $10-20 \mathrm{~K}$ rather than $100 \mathrm{~K}$ ) (Tielens et al. 1991). Chemical studies must include the abundant molecules $\mathrm{N}_{2}$ and $\mathrm{O}_{2}$ both of which form ices (Tielens et al. 1991). Unfortunately these molecules are undetectable since in the solid phase neither molecule is infrared active and in the gas phase both have only very weak infrared quadrupole spectra.

\subsection{Oxygen-Rich Circumstellar Shells}

As indicated by circumstellar chemistry models, the more interesting objects for the infrared spectroscopist are the carbon-rich circumstellar shells. The oxygenrich circumstellar shells are dominated by the spectrum of $\mathrm{H}_{2} \mathrm{O}$. Water has a rich, complex gas-phase spectrum. As early as the 1960's work by Kuiper (1963) and Johnson et al. (1968) showed that the photospheric spectrum of cool M-giants was dominated by water bands in the $1-2.5 \mu \mathrm{m}$ region. Chemical equilibria calculations show that for $\mathrm{C}<\mathrm{O}$ water is a dominant constituent at temperatures less than about $3000 \mathrm{~K}$ (Jørgensen this volume). Only for effective temperatures below about $1800 \mathrm{~K}$ at relatively high gravities do other polyatomic molecules $\left(\mathrm{CH}_{4}\right)$ start to compete with $\mathrm{H}_{2} \mathrm{O}$ (Tsuji this volume). Hinkle \& Barnes (1979) noted that in oxygen-rich Mira variables water lines could be seen originating in the upper photosphere as well as in a $\sim 1000 \mathrm{~K}$ region. The $\sim 1000 \mathrm{~K}$ region appears to be the inner circumstellar shell (Hinkle et al. 1994). Unfortunately, water has such a complex spectrum that it is difficult to analyze on a line by line basis. In spite of significant efforts by several groups (see for example CamyPeyret et al. 1977) literally thousands of unidentified water lines remain in the infrared spectra of astronomical sources. Mozurkewich et al. (1987) discovered a rich circumstellar water spectrum in the $R V$ Tau star $R$ Sct. In the case of $\mathrm{R}$ Sct the star is not obscured but the photosphere is too hot for water lines. While the above references are to $2 \mu \mathrm{m}$ region spectra, water contributes lines throughout the infrared into the sub-millimeter and millimeter wavelength regions. Strong bands are obvious in M-giant photospheric spectra in the $3-4 \mu \mathrm{m}$ region (Ridgway et al. 1984) and are the dominant feature of $10 \mu \mathrm{m}$ sunspot spectra (Wallace \& Livingston 1993). Microwave water masers originating from oxygen-rich circumstellar shells are well known.

The ubiquitous $\mathrm{CO}$ molecule also contributes spectral features in oxygen-rich circumstellar shells. Circumstellar CO fundamental lines are obvious in the 4.6 $\mu \mathrm{m}$ spectra of $\mathrm{M}$ stars and $2.3 \mu \mathrm{m}$ first overtone lines can have a circumstellar contribution. Weak circumstellar $\mathrm{H}_{2}$ lines may be observed in the $2 \mu \mathrm{m}$ region in M-type giants (Hinkle et al. 1994). Circumstellar $\mathrm{SiO}$ is observable in the 4 and $10 \mu \mathrm{m}$ region (Geballe et al. 1979; Tsuji et al. 1994).

Finally, absorption bands caused by ices and silicate grains are major contributors to infrared spectra of oxygen-rich objects. A detailed discussion of these topics is beyond the scope of the present review, and the reader is referred to e.g. Allamandola 1984; Allamandola \& Sandford 1988; Tielens \& Allamandola 1987. 
See also the reviews by Alexander \& Ferguson and by Sedlmayr in this volume. Linked with the above discussion of gas phase $\mathrm{H}_{2} \mathrm{O}$, water ice has long been known to be the origin of the $3.08 \mu \mathrm{m}$ continuous absorption band seen toward very obscured late-type stars and in spectra of embedded sources in dense clouds (Gillett \& Forrest 1973). Recent work by Smith et al. (1988) indicates that this band results from water frozen in an amorphous layer onto silicate grains. This is an interesting and very well documented case of freeze out of a gas phase constituent of the circumstellar shell onto a grain mantle.

\section{The Future of Infrared Spectroscopy}

Until recently infrared detectors were available only in single pixel format and were plagued with very high inherent noise levels. Recording narrow spectral elements of an astronomical source one by one with such a detector was virtually impossible. Fourier transform spectroscopy (FTS), which was used for most of the observations reported here, features a natural multiplexing strategy which encodes many spectral elements on a single detector. As long as detector noise dominates, FTS offers a gain over a single channel device on order of the number of spectral resolution elements. More or less by chance, FTS also offers a number of wonderful instrumental features: no slit; continuously adjustable and unlimited spectral resolution; well understood instrumental line profile; very large spectral coverage; very high throughput; no scattered light; accurately known, internally calibrated frequency scale without a dispersion relation; noise dominated wavelength precision; inherently photometric spectra. Unfortunately FTS, as implemented for high resolution spectroscopy, has the severe limitation that it lacks the sensitivity to reach 'faint' sources.

Infrared arrays with dimension of $256 \times 256$ pixels are now widely available in a number of materials ( $\mathrm{InSb}, \mathrm{HgCdTe}, \mathrm{PtSi}$ ). While infinite increase in size is not possible, arrays a factor of 2-4 larger are likely. These arrays offer a large number of pixels with each pixel having very low noise. The array detectors are integrating detectors, rather than continuous readout as were the single element detectors of the past. Assuming high quality devices of both types (noise equivalent power [NEP, see e.g. Wolfe and Zissis 1989] for the single element detector of $10^{-18} \mathrm{watt} / \sqrt{\mathrm{Hz}}$ and readout noise for the integrating detector of 30 $\mathrm{e}^{-}$with negligible dark current), then the signal to noise (S/N) and integration time in seconds $(t)$ are related by:

$$
\log _{10}(\text { relative } \mathrm{S} / \mathrm{N} \text { [integrating/analog] })=0.5 \log _{10}(\mathrm{t})+0.5
$$

(Ridgway \& Hinkle 1992).

From equation 1, we conclude that a grating spectrometer with an infrared array will be on the order of $100 \times$ more sensitive than an FTS. Generally, a spectrograph that takes advantage of infrared arrays is cryogenic. Infrared arrays become background limited in warm spectrographs at wavelengths longer than about $1.8 \mu \mathrm{m}$. Several cryogenic spectrographs have now been built to 
use infrared arrays (CSHELL at IRTF and CGS4 at UKIRT). These spectrographs offer two pixel resolution of about 20000, with 40000 at the limiting end of their performance. At NOAO, we have been working on a resolution 70,000-100,000 spectrograph intended for use on the 2.1 and 4 meter telescopes. This spectrograph will have obvious applications in circumstellar and interstellar spectroscopy.

\section{References}

Alcock C., Ross R.R., 1986, Astrophys. J., 310, 838

Allamandola L.J., 1984, in Galactic and Extragalactic Infrared Spectroscopy, ed. M.F. Kessler, J.P. Phillips (Dordrecht:Reidel), p. 5

Allamandola L.J., Sandford S.A., 1988, in Dust in the Universe, ed. M.E. Bailey, D.A. Williams (Cambridge: Cambridge Univ. Press), p. 229

Bernat A.P., 1981, Astrophys. J., 246, 184

Bernat A.P., Hall D.N.B., Hinkle K.H., Ridgway S.T., 1979, Astrophys. J., 233, L135

Bernat A.P., Lambert D.L., 1976, Astrophys. J., 210, 395

Bernath P.F., 1992, in Astronomical Infrared Spectroscopy ed. S. Kwok (ASP Conf. Series Vol. 41), p. 251

Bernath P.F, Hinkle K.H., Keady J.J., 1989, Science, 244, 562

Betz A.L., McLaren R.A., Spears D.L., 1979, Astrophys. J., 229, L97

Bieging J.H., Tafalla M., 1993, Astron. J., 105, 576

Brown P.D., Charnley S.B., Miller T.J., 1988, Mon. Not. Royal Astron. Soc., 231, 409 Camy-Peyret C., Flaud J.M., Toth R.A., 1977, J. Mol. Spec., 67, 117

Clegg R.E.S., Lambert D.L., Hinkle K.H., 1982, Mon. Not. Royal Astron. Soc., 201, 95

Danchi W.C., Bester M., Degiacomi C.G., McCullough P.R. Townes C.H., 1990, Astrophys. J., 359, L59

Dominik C., Gail H.-P., Sedlmayr E., Winters J.M., 1990, Astron. Astrophys., 240, 365

Dupree A.K., 1986, Ann. Rev. Astron. Astrophys., 24, 377

Frenklach M., Feigelson E.D., 1989, Astrophys. J., 341, 372

Frum et al., 1991, Chem. Phys. Lett., 176, 505

Geballe T.R., Lacy J.H., Beck S.C., 1979, Astrophys. J., 230, L47

Genzel R., 1992, in The Galactic Interstellar Medium, ed. D. Pfenniger, P. Bartholdi (Springer-Verlag:Berlin)

Gillett F.C., Forrest W.J., 1973, Astrophys. J., 179, 483

Glassgold A.E., Huggins P.J., 1987, in The M-type Stars, ed. H. Johnson, F. Querci (NASA SP-492)

Glassgold A.E., Lucas R., Omont A., 1986, Astron. Astrophys., 157, 35

Goldberg L., 1987, in The M-type Stars, ed. H. Johnson, F. Querci (NASA SP-492)

Goldh aber D.M., Betz A.L., 1984, Astrophys. J., 279, L55

Goldhaber D.M., Betz A.L., Ottusch J.J., 1987, Astrophys. J., 314, 356

Harvey P.M., Lester D.F., Brock D., Joy M., 1991, Astrophys. J., 368, 558

Hebden J.C., Eckart A., Hege E.K., 1987, Astrophys. J., 314, 690

Hinkle K.H., Barnes T., 1979, Astrophys. J., 227, 923

Hinkle K.H., Bernath P., 1992, in Astronomical Infrared Spectroscopy, ed. S. Kwok (ASP Conf. Series Vol. 41), p. 125

Hinkle K.H., Keady J.J., Bernath P.F., 1988, Science, 241, 1319 
Hinkle K.H., Ridgway S.T., Martin C., 1994a, Astrophys. J., submitted

Hinkle K.H., Keady J.J., Sada, P., 1994b, in preparation

Howe D.A., Millar T.J., 1990, Mon. Not. Royal Astron. Soc., 244, 444

Johnson H.L., Coleman I., Mitchell R.I, Steinmetz D.L., 1968, Comm. Lunar Planet. Lab., 7, 83 (No. 113)

Jones T.W., Ney E.P., Stein W.A., 1981, Astrophys. J., 250, 324

Jørgensen U.G., 1990, Astron. Astrophys., 232, 420

Jørgensen U.G., 1992, Rev. Mex. Astron. Astrof., 23, 49

Jura M., 1986, Astrophys. J., 303, 327

Kastner J.H., 1992, Astrophys. J., 401, 337

Keady J.J., Hall D.N.B., Ridgway S.T., 1988, Astrophys. J., 326, 832

Keady J.J., Hinkle K.H., 1988, Astrophys. J., 331, 539

Keady J.J., Ridgway S.T., 1993, Astrophys. J., 406, 199

Kratschmer W., Lamb L.D., Fostiropoulos K., Huffman D.R., 1990, Nature, 347, 354

Kroto H.M., Heath J.R., O'Brien S.C., Curl R.F., Smalley R.E., 1987, Astrophys. J., 314, 352

Kuiper G.P., 1963, Comm. Lunar Planet. Lab., 1, 179 (No. 23)

Lacy J.H., Carr J.S., Evans N.J., Bass F., Achtermann J.M., Arens J.F., 1991, Astrophys. J., 376, 556

Lafont S., Lucas R., Omont A., 1982, Astron. Astrophys., 106, 201

Latter W.B., 1991, Astrophys. J., 377, 187

Léger A., d'Hendecourt L., Boccara N. ed., 1987, Polycyclic Aromatic Hydrocarbons and Astrophysics, (Dord recht:Reidel)

Mamon G.A., Glassgold A.E., Omont A., 1987, Astrophys. J., 323, 306

Mauron N., Fort B., Querci F., Dreux M., Fauconnier T., Lamy P., 1984, Astron. Astrophys., 130, 341

Mozurkewich D., Gehrz R.D., Hinkle K.H., Lambert D.L., 1987, Astrophys. J., 314, 242

McCabe E.M., Connon Smith R., Clegg R.E.S., 1979, Nature, 281, 263

Moran J.M., Ball J.A., Predmore C.R., Lane A.P., Huguenin G.R., Reid M.J., Hansen S.S., 1979, Astrophys. J., 231, L67

Morris M., Guilloteau S., Lucas R., Omont A., 1987, Astrophys. J., 321, 888

Nejad L.A.M., Millar T.J., 1987, Astron. Astrophys., 183, 279

Nejad L.A.M., Millar T.J., Freeman A., 1984, Astron. Astrophys., 134, 129

Olofsson H., 1992, in Mass Loss on the $A G B$ and Beyond, in press

Olofsson H., Eriksson K., Gustafsson B., Carlstrom U., 1993, Astrophys. J. Suppl., 87, 267

Omont A., 1985, in Mass-Loss from Red Giants, ed. M. Morris, B. Zuckerman (Dordrecht: Reidel), p. 269

Omont A., Lucas R., Morris M., Guilloteau S., 1993, Astron. Astrophys., 267, 490

Ridgway S.T., Hinkle K.H., 1992, in High Resolution Spectroscopy with the VLT, ed. M.-H. Ulrich (ESO Conf. \& Workshop Proceedings 40), p. 213

Ridgway S.T., Carbon D.F., Hall D.N.B., 1978, Astrophys. J., 225, 138

Ridgway S.T., Carbon D.F., Hall D.N.B., Jewell J., 1984, Astrophys. J. Suppl., 54, 177

Sahai R., Wannier P.G., 1985, Astrophys. J., 299, 424

Scalo J.M., Ross J.E., 1976, Astron. Astrophys., 48, 219

Smith R.G., Sellgren K., Tokunaga A.T., 1988, Astrophys. J., 334, 209

Tielens A.G.G.M., Allamandola L.J., 1987, in Interstellar Processes, ed. D. Hollenbach, H. Thronson (Dordrecht:Reidel), p. 397 
Tielens A.G.G.M., Tokunaga A.T., Geballe T.R., Baas F., 1991, Astrophys. J., 381, 181

Tsuji T., 1964, Ann. Tokyo Astron. Obs., 2nd Ser.,9, 1

Tsuji T., 1973, Astron. Astrophys., 23, 411

Tsuji T., Ohnaka K., Hinkle K.H., Ridgway S.T., 1994, Astron. Astrophys., submitted. Turner B.E., 1991, Astrophys. J., 376, 573

Wallace L., Livingston W., 1993, in preparation

Wannier P.G., Sahai R., 1987, Astrophys. J., 319, 367

Wiedemann G.R., Hinkle K.H., Keady J.J., Deming D., Jennings D.E., 1991, Astrophys. $J ., 382,321$

Wolfe W.L., Zissis G.J. eds. 1989 The Infrared Handbook, (Environmental Research Institute of Michigan, Ann Arbor, Michigan)

Zuckerman B., 1980, Ann. Rev. Astron. Astrophys., 18, 263 